\title{
LABORATORY INFRARED SPECTROSCOPY OF PAHS
}

\author{
J. Oomens ${ }^{1,2}$
}

\begin{abstract}
The hypothesis that polyaromatic molecules are the carriers of the infrared interstellar emission bands has spurred the laboratory spectroscopy of this class of molecules. Here we will give an overview of the infrared spectroscopic methods that have been applied over the past two decades to investigate the IR spectra of PAHs, their ions and related species.
\end{abstract}

\section{Introduction}

One of the earlier comparisons of a spectrum of the unidentified infrared (UIR) bands - from the Orion Bar region - and an experimental laboratory spectrum of a sample of polyaromatic species was reported by Allamandola et al. (1985), who used a low-resolution Raman spectrum of a car exhaust sample as the reference PAH spectrum. In their seminal work, Léger \& Puget (1984) used a calculated emission spectrum of coronene as a reference spectrum to explain the UIR bands. These examples illustrate the very limited availability of laboratory spectroscopic data on PAH species at the time the PAHs were first proposed as UIR band carriers. Although some pellet and solution-phase spectra of PAHs had been obtained with grating instruments (Colangeli et al. 1992), the PAH hypothesis inspired spectroscopists around the World to record infrared PAH spectra under better defined and more astrophysically relevant conditions.

The interstellar emission spectra are believed to be carried by gas-phase PAH species in complete isolation. Except in highly irradiated environments, their rotational temperature is believed to be below $100 \mathrm{~K}$ (Ysard \& Verstraete 2010), while the vibrational temperature is estimated to be on the order of $1000 \mathrm{~K}$ after the absorption of an interstellar UV photon and subsequent internal conversion releasing the UV photon energy into the vibrational manifold of the electronic ground state. Finally, spectra recorded in emission rather than in absorption are astrophysically more relevant.

\footnotetext{
1 FOM Rijnhuizen, Edisonbaan 14, 3439MN Nieuwegein, The Netherlands

2 van't Hoff Institute for Molecular Sciences, University of Amsterdam, Amsterdam, The Netherlands
} 
In this contribution, we will briefly review the experimental methods that have been applied over the past 25 years to obtain infrared spectra of isolated PAHs.

\section{Neutral PAH infrared spectroscopy}

To reduce the effects of the medium on the IR spectra recorded (Flickinger et al. 1991; Moutou et al. 1996), an inert cryogenic Ar matrix was used instead of the salt pellets or solution (typically $\mathrm{CCl}_{4}$ ). This also enabled spectroscopists to obtain spectra at low temperature, which is more relevant to the conditions of dense clouds. But even the rare gas matrix can influence the appearance of the IR spectra (Joblin et al. 1994) and hence gas-phase absorption spectra were recorded, although this required heating the samples in an oven as a consequence of the low vapor pressure of the PAHs. The use of molecular beam methods combined with laser spectroscopy allowed spectroscopists to obtain low-temperature gas-phase absorption spectra. It was further realized that emission spectra, as observed from the ISM, may be different from absorption spectra as recorded with the use of (commercial) spectrometers. Various spectroscopists thus set out to record gas-phase IR emission spectra, which perhaps provide the most astrophysically relevant neutral $\mathrm{PAH}$ spectra to date.

\subsection{Matrix isolation spectroscopy}

In matrix isolation, a host molecule (the PAH in this case) and an inert guest (often the rare gas atoms $\mathrm{Ar}$ or $\mathrm{Ne}$ ) are simultaneously condensed onto an optical window, which is kept at a cryogenic temperature in a cryostat. The technique has been widely applied to study reactive species, which are stabilized by the low temperature and inert environment. As a consequence of the low temperature and the quenching of rotational degrees of freedom in the matrix, IR spectra of matrix-isolated PAHs feature sharp absorption bands with FWHM linewidths on the order of $1 \mathrm{~cm}^{-1}$. An extensive database of infrared PAH spectra recorded in cryogenic Ar matrices has been collected by the NASA Ames group ${ }^{1}$.

Recent applications include the study of nitrogen containing PAHs (Mattioda et al. 2003), which have been suggested to provide a better match for the interstellar $6.2 \mu \mathrm{m}$ emission band (Hudgins et al. 2005; Peeters et al. 2002), and of complexes of PAHs and atomic Fe, which have been suggested to be stable in interstellar clouds (Klotz et al. 1996; Wang et al. 2007). The method has also been applied to record PAH spectra in the far-infrared range of the spectrum (Mattioda et al. 2009), where very molecule-specific vibrational modes - the socalled "drumhead" modes - are located.

\footnotetext{
${ }^{1}$ See www. astrochem.org/pahdb
} 


\subsection{Gas-phase absorption spectroscopy}

While the matrix isolation spectra provide high resolution spectra for a large number of PAH molecules, the influence of the matrix on the frequencies and, in particular, intensities remains unknown (Joblin et al. 1994). Gas-phase spectra were considered to be more relevant from an astrophysical point of view and could, moreover, shed more light on possible matrix effects. Except for the smallest members of the family, however, the vapor pressure of PAHs is too low to record direct absorption spectra.

This issue was addressed by Joblin et al. (1994), who set up a heatpipe oven inside an FTIR spectrometer to record gas-phase IR absorption spectra of PAHs up to the size of ovalene $\left(\mathrm{C}_{32} \mathrm{H}_{14}\right)$. This study indicated that while gas-phase and matrix isolated band positions reproduce to within about $1 \%$, band intensities can change by up to a factor of five. While the high temperatures needed to vaporize the molecules lead to substantially larger bandwidths and may appear to be astrophysically unrealistic, interstellar emission occurs from excited PAH molecules which are estimated to possess vibrational temperatures on the order of $1000 \mathrm{~K}$ (Joblin et al. 1995). Moreover, spectra recorded at different temperatures of the oven yield direct information on the anharmonic frequency shifts of vibrational bands (Pirali et al. 2009), which are important in modelling IR emission spectra (Cook \& Saykally 1998) and which are hard to estimate computationally for large systems.

Gas-phase spectra recorded at low temperature are expected to give the best values for the intrinsic molecular band positions, free from shifting due to anharmonic interactions or matrix effects. To obtain such low-temperature spectra for low-vapor pressure compounds, molecular beam techniques can be applied. Here, low rovibrational temperatures are achieved by expansion cooling of the molecules in a supersonic beam of rare-gas atoms. Typical rotational temperatures are on the order of $10 \mathrm{~K}$; vibrational cooling can be less efficient. The low densities of molecules in these beams require the application of tunable lasers to obtain (infrared) spectra of the jet-cooled PAHs. Piest et al. (2001) applied such methods at the infrared free electron laser facility FELIX (Oepts et al. 1995) to obtain IR spectra of small PAH molecules, such as phenanthrene.

\subsection{Infrared emission spectra}

Interstellar UIR spectra are observed as emission spectra rather than as absorption spectra and hence, laboratory emission spectra are desirable. One of the main difficulties in these experiments is to distinguish the PAH IR fluorescence from the blackbody emission of the environment. Early experiments on UV excited PAH species therefore only recorded spectra in the $3 \mu \mathrm{m}$ spectral range (Shan et al. 1991; Williams \& Leone 1995). Thermally heated gas-phase PAH samples were used to increase the number density of emitting molecules so that the infrared PAH emission could be better distinguished from the background allowing various groups to record spectra at higher resolution (Pirali \& Vervloet 2006) and further 
into the infrared (Joblin et al. 1995). The evaporated PAHs, however, form a sample in thermal equilibrium, while UV-excited PAHs combine low rotational excitation with high vibrational excitation, a situation that more closely resembles the assumed conditions in the ISM. Perhaps the most sophisticated emission spectroscopy experiments have been reported by the group of Saykally, who used a cryogenically cooled grating spectrometer to greatly suppress the blackbody background radiation, in combination with an extremely sensitive IR detector (Cook et al. 1998, 1996). Thus, emission spectra of UV excited PAHs were recorded over the entire IR range from 3 to $15 \mu \mathrm{m}$.

\section{IR spectroscopy of ionic PAH species}

One of the main conclusions of the emission spectra of Cook et al. (1996) was that neutral PAH species would not be able to explain the UIR emission features and the presence of a substantial fraction of ionized PAHs was invoked. In fact, already early on in the history of the PAH hypothesis it was realized that ionized PAHs may play an important role because of the low ionization potentials of PAHs combined with the UV pump fields that are necessary to induce IR emission (Allamandola et al. 1989). An important difference between the spectra of neutral and cationic PAHs was first revealed by quantum-chemical calculations on the naphthalene radical cation by Pauzat et al. (1992); see also the contribution by Pauzat elsewhere in this volume.

These considerations inspired various laboratory spectroscopists to record spectra of ionized PAH species, again preferably under astrophysically relevant conditions. Because of their mutual repulsion and inherently low gas-phase number densities, direct absorption spectra of (mass-selected) gas-phase ions are difficult, if not impossible, to record. Matrix isolation spectroscopy offers a way around this problem, but also gas-phase "action spectroscopy" schemes have more recently been applied. Here we discuss the different methods applied to date and compare their merits and limitations.

\subsection{Matrix isolation spectroscopy}

Immediately following the computations of Pauzat et al. (1992), the group of Vala at the University of Florida set out to record the naphthalene cation spectrum using a modified matrix isolation set up (Szczepanski et al. 1992). The effusive naphthalene beam, which is co-deposited onto the $12-\mathrm{K} \mathrm{BaF}_{2}$ substrate with an Ar beam, was intercepted by an electron beam with electron energies on the order of $50-200 \mathrm{eV}$. The Ar sample was premixed with $\mathrm{CCl}_{4}$, which acts as an electron acceptor, reducing the re-neutralization of the naphthalene cations once in the matrix. The IR spectrum of the matrix was then recorded using an FTIR spectrometer. One possible pitfall of this method is the possible recombination or reactivity of the naphthalene cation in the matrix. Observed IR bands of neutral naphthalene are therefore subtracted. Moreover, to assign newly observed IR bands to the cation of interest, their intensity is typically correlated with those of 
known bands in a UV/vis spectrum recorded from the same matrix (Szczepanski \& Vala 1991).

The experimental naphthalene cation spectrum confirmed the computational results and showed definitively that the relative intensities of the different families of vibrational modes undergo major changes upon ionization. Soon after this first PAH cation spectrum, IR matrix isolation spectra of a whole series of cationic PAHs were reported mainly by the Florida (Szczepanski \& Vala 1993a) and NASA Ames (Hudgins \& Allamandola 1995a,b; Hudgins et al. 1994) groups. These spectra provided the first experimental evidence that ionized PAHs may be abundant in the ISM (Allamandola et al. 1999; Szczepanski \& Vala 1993b). In addition, these spectra provided the experimental verification for various quantum-chemical studies on the IR properties of ionized PAHs. For the vast majority of the PAH species that have been studied, a good correlation was found between experimental frequencies (and intensities) and values computed at the density functional level of theory (see e.g. Langhoff (1996)). This provides confidence in the spectra calculated for species that are experimentally not easily accessible, such as very large PAHs (Bauschlicher et al. 2008; Malloci et al. 2007).

Despite the success of matrix isolation spectroscopy and the ever growing database of spectra, the possible unknown effects of the matrix on band frequencies and intensities made that the desire to record true gas-phase $\mathrm{PAH}$ ion spectra remained. The main hurdle to record such spectra were the extremely low densities in which gas-phase ions are typically produced as a consequence of their mutual Coulombic repulsion. Spectroscopic methods based on conventional direct absorption techniques are therefore generally not applicable to (mass-selected) molecular ions. Since the 1980's, however, infrared laser-based methods became increasingly successful in recording gas-phase molecular ion spectra (Duncan 2000).

\subsection{Messenger atom spectroscopy}

One of the most influential laser-based methods was originally introduced by Y.T. Lee and coworkers and became known as "messenger-atom" spectroscopy (Lisy 2006; Okumura et al. 1985, 1990). The method is based on a supersonic expansion in which ionized molecular species form weakly bound complexes with neutral molecules or atoms, which are mass-selected typically in a quadrupole mass selector (QMS) or sector magnet. Upon excitation with an IR laser, the complex undergoes pre-dissociation and the now bare molecular ion is detected in a mass analyzer, typically a time-of-flight (TOF) or quadrupole mass spectrometer. In all such combinations of tandem mass spectrometry and laser spectroscopy, monitoring the intensity of the mass peak of the product molecular ion as a function of the IR wavelength then generates an IR spectrum of the ionic complex. While the entire ionic complexes have been the subject of many studies, it was also realized that the neutral partner in the complex could be used solely as the detector of IR photon absorption by the molecular ion, as signalled by its detachment from the ion, hence the term "messenger". Clearly in this case, the messenger was to have an as small as possible influence on the ionic species of interest - or in the words of Y.T. Lee, 
it was to behave as a spy (Okumura et al. 1990) - and hence, the use of rare gas atoms rather than small molecules became popular. Messenger atom spectroscopy was for instance used to record the IR spectra of several ionized aromatic systems, particularly benzene and various of its ring-substituted derivatives (Solcà \& Dopfer 2004).

The early studies based on messenger spectroscopy focused particularly on the hydrogen stretching vibrations in the $3 \mu \mathrm{m}$ wavelength range, since tunable radiation can be generated in this range by various methods, including frequency mixing of the output of a tunable dye laser in birefringent crystals. Towards the late 1990 's, new widely tunable laser sources became available that covered the astrophysically interesting wavelength range roughly between 6 and $16 \mu \mathrm{m}$. Novel birefringent materials allowed non-linear frequency down-conversion methods to penetrate further into the IR (Bosenberg \& Guyer 1993). Moreover, tunable infrared free electron lasers (O'Shea \& Freund 2001), where radiation is generated by a relativistic beam of electrons injected into a periodic magnetic field structure (wiggler), entered the realm of spectroscopy.

Using the free electron laser at the FELIX facility, Meijer and coworkers recorded the gas-phase IR spectra of the naphthalene (Piest et al. 1999a) and phenanthrene (Piest et al. 2001) cations using a variation of the messenger-atom spectroscopy method (Piest et al. 1999b). Van der Waals clusters of the PAH and a rare gas atom (Ar in most cases) were produced in a pulsed molecular beam expansion and they were resonantly ionized using a two-color resonance enhanced multiphoton ionization (REMPI) scheme. The thus formed intact $\mathrm{PAH}^{+}-\mathrm{Ar}$ complexes were then irradiated by the output of the free electron laser, which was tuned in wavelength across the entire 6 to $16 \mu \mathrm{m}$ range. IR absorption was signalled as the appearance of the bare $\mathrm{PAH}^{+}$ion in a TOF mass spectrometer. Since the influence of the weakly bound Ar atom is assumed to be negligible, the spectra, which feature a resolution of just a few $\mathrm{cm}^{-1}$, can be considered as being due to the cold and isolated PAH cation.

The group of Duncan recently recorded IR spectra of protonated benzene (Douberly et al. 2008) and naphthalene (Ricks et al. 2009) using an optical parametric oscillator (OPO) source that provides mJ pulse energies down to wavelengths of about $10 \mu \mathrm{m}$. Argon tagged protonated molecules are produced in the expansion from a pulsed discharge nozzle and mass-selected in the first arm of a reflectron TOF mass spectrometer. The ions are then exposed to the IR radiation in the turning point of the reflectron stack and mass analysis is accomplished in the second arm of the reflectron. Also here, detachment of the messenger (Ar) is used to signal IR absorption by the protonated PAH, giving the spectrum of the ionized PAH at a resolution of a few $\mathrm{cm}^{-1}$.

\subsection{IR multiple photon dissociation spectroscopy}

In the 1980's, various groups explored the possibilities of gas-phase infrared ion spectroscopy by wavelength selective multiple photon dissociation of ions in storage mass spectrometers using powerful line-tunable $\mathrm{CO}_{2}$-lasers (see Eyler 2009 for a 
recent review). Although a variety of ionic systems was successfully studied, the limited tuning range of the $\mathrm{CO}_{2}$-laser (roughly $9-11 \mu \mathrm{m}$ ) severely impeded the practical applicability of this method for spectroscopic purposes. More recently, the infrared pulse energies produced by free electron lasers were shown to be high enough to induce photodissociation of vapor-phase PAH cations (Oomens et al. 2000). Installation of an ion trap time-of-flight mass spectrometer at the free electron laser facility FELIX thus allowed Oomens et al. (2003) to record IR multiple photon dissociation (IRMPD) spectra for a large number of mass selected ionic PAHs. The PAHs are vaporized in an oven and effuse to the center of a quadrupole ion trap, where they are non-resonantly ionized by a UV laser (excimer). Possible UV-induced fragments are mass-selectively removed from the trap and the isolated parent ions are irradiated with the FEL beam. The IR induced fragments and the remaining parent ions are then mass-analyzed in a TOF spectrometer, so that an IRMPD spectrum can be reconstructed by plotting the fragment yield as a function of the FEL wavelength.

Dissociation thresholds for $\mathrm{PAH}$ ions are typically in the $6-8 \mathrm{eV}$ range, which requires the absorption of tens to hundreds of IR photons. In polyatomic molecules of the size of PAHs, infrared multiple photon excitation typically proceeds via rapid redistribution of the vibrational energy from the excited mode into the bath formed by all vibrations of the molecule. Intramolecular vibrational redistribution (IVR) thus allows the molecule to sequentially absorb multiple photons on the same vibrational transition, because the upper level is constantly being deexcited by quenching to the bath states (Black et al. 1977; Eyler 2009; Grant et al. 1978). IVR-mediated multiple photon excitation thus avoids vibrational ladder climbing, which is an inefficient process due to the anharmonicity bottleneck. The multiple-photon nature of this spectroscopic technique is reflected in the shape of the spectral features observed, most notably from the increased bandwidth as compared to messenger-atom or matrix isolation spectroscopy. The PAH ion spectra recorded at FELIX using this method display FWHM bandwidths on the order of $30 \mathrm{~cm}^{-1}$ (Oomens et al. 2000).

At the free electron laser facilities CLIO and FELIX, the FEL beam has now been coupled to powerful tandem mass spectrometers, so that a variety of ionic PAH-related species can be spectroscopically studied. The ions can be produced by a variety of ion sources, including electrospray ionization (ESI) and laser desorption/ablation methods. Much recent interest has been on protonated PAH species including protonated regular PAHs (Knorke et al. 2009; Lorenz et al. 2007; Vala et al. 2009b) and protonated N-containing PAHs (Alvaro Galué et al. 2010; Vala et al. 2009a). Clear differences are observed in the spectra of (even-electron) protonated species and (odd-electron) radical cation species. PAHs have long been suggested to efficiently bind to astrophysically abundant metal atoms or ions (Joalland et al. 2009; Klotz et al. 1996) and such species are now being investigated spectroscopically using IRMPD spectroscopy (Simon et al. 2008; Szczepanski et al. 2006). 


\subsection{IR emission spectroscopy}

As mentioned in Section 2.3 for neutral PAH species, IR spectra recorded in emission instead of absorption, as is more common, have the advantage that they mimick better the interstellar situation, where the UIR bands are observed in emission as well. However, the difficulty in recording emission spectra in the astrophysically most relevant $6-16 \mu \mathrm{m}$ range lies mainly in distinguishing the weak infrared PAH fluorescence from the blackbody radiation of the environment. This is an even more daunting task for ionic species as compared to neutrals, as the ion densities are intrinsically much lower. Nonetheless, the Saykally group at Berkeley managed to adapt their cryogenically cooled spectrometer so that it could be used to record emission spectra of gas-phase ionic PAHs (Kim \& Saykally 2003). To this end, the spectrometer is coupled to an electron impact ion source, which produces high ion currents of more than $6 \mu \mathrm{A}$. Electron impact with approximately $70 \mathrm{eV}$ electrons not only ionizes the molecules, but also induces vibronic excitations that are comparable to UV excitation; hence, the IR emission from the ions is assumed to be similar to that from UV excited ions as occurs under interstellar conditions. Using a quadrupole deflector, the ions are injected into a reflectron, which is placed in front of the entrance slit of the spectrometer. A reflectron is used to increase the residence time of the ions in the viewing region of the spectrometer. All ion optics in the line of sight of the spectrometer need to be cryogenically cooled while being electrically isolated, which constituted one of the main design challenges (Kim \& Saykally 2003).

IR emission spectra were reported for the pyrene cation $\left(\mathrm{C}_{16} \mathrm{H}_{10}^{+}\right)$and several dehydrogenated derivatives (Kim \& Saykally 2002; Kim et al. 2001). The pyrene cation spectrum showed a good correspondence with matrix isolation and IRMPD spectra of the pyrene cation. Additional bands observed in the emission spectrum could be attributed to partly dehydrogenated fragments of the pyrene cation.

\section{Experimental methods compared}

A discussion of the various experimental methods applied for PAH spectroscopy inevitably raises the question as to which of the techniques is superior. This question is, however, not easily answered: each method has its strong and weak points.

Of all spectroscopic methods discussed here, IR emission spectroscopy yields spectra under conditions that are arguably most closely mimicking those of the interstellar PAHs. The gaseous PAHs are electronically excited to energies in the range of the interstellar conditions. The effects of vibrational anharmonicity (Barker et al. 1987; Pirali et al. 2009) are naturally incorporated into the observed emission bands. This is clearly an advantage over spectra obtained using absorption spectroscopy since for a true comparison to interstellar emission spectra, one would have to convolute the bands using an emission model including the vibrational anharmonicities (Cook \& Saykally 1998; Pech et al. 2002), which are however largely unknown. However, the experiments are extremely challenging, 
particularly for ionic PAHs. The method requires a cryogenically cooled IR spectrometer with a very sensitive IR photon detector; for the ions, a high ion current source is required as well. Thus far, only the group of Saykally have reported IR emission spectra of PAH ions in the $5-15 \mu \mathrm{m}$ spectral range, and only very few spectra (of pyrene and some of its dehydrogenated fragments) have been reported.

Quite in contrast, matrix isolation spectroscopy is experimentally most readily implemented. Indeed, most PAH ion spectra reported to date have been obtained using this method. We may also note that variants of this method are currently being applied to study the spectroscopy and (photo-)chemistry of PAHs and other species in interstellar ices (Bernstein et al. 2007; Bouwman et al. 2009; Klotz et al. 1996). Matrix isolation spectra feature narrow bandwidths induced by the low temperature and the quenching of rotational motion in the rare gas matrix. Experimentally, matrix isolation spectroscopy may suffer from unknown effects of the matrix on the spectrum; in the infrared spectra, it appears that particularly relative intensities are affected (Joblin et al. 1994). Moreover, there is some uncertainty as to the exact nature of the species trapped in the matrix, particularly upon electron or UV irradiation, and correlation measurements are usually required to assign bands to specific species (Szczepanski \& Vala 1991). This problem may be partly circumvented by mass-selective deposition of ions created ex-situ, see e.g. Fulara et al. (1993).

PAH ion spectra obtained using messenger atom spectroscopy have arguably provided the most reliable linear absorption spectra thus far. Bandwidths of just a few $\mathrm{cm}^{-1}$ have been obtained for PAH species that are estimated to be expansion cooled to temperatures on the order of $20 \mathrm{~K}$. These methods require the availability of IR laser sources that are continuously tunable in the $5-15 \mu \mathrm{m}$ spectral range and thus far free electron lasers and optical parametric oscillators have been used. Moreover, the PAHs need to be entrained in a seeded molecular beam expansion to form the weakly bound PAH-rare gas complexes, which has thus far only been possible for the smallest PAHs naphthalene (Piest et al. 1999a; Ricks et al. 2009) and phenanthrene (Piest et al. 2001).

IRMPD spectroscopy has the advantage that it can be conveniently coupled to (commercial) tandem mass spectrometers, providing access to many species beyond regular $\mathrm{PAH}$ ions. A tunable laser source with high output powers is required to induce fragmentation of the ions and thus far, mainly the free electron lasers FELIX in The Netherlands (Oomens et al. 2000) and CLIO in France (Lorenz et al. 2007) have been used for these studies. The spectral band shapes observed are clearly influenced by the process of multiple photon excitation (Oomens et al. 2003) resulting in FWHM bandwidths of typically $30 \mathrm{~cm}^{-1}$ or more, which tends to produce unresolved spectra for larger, more irregularly shaped species. Nonetheless, PAH ion spectra up to the size of coronene $\left(\mathrm{C}_{24} \mathrm{H}_{12}\right)$ have been reported (Knorke et al. 2009; Oomens et al. 2001; Simon et al. 2008). In addition, one may note that the broadening of bands is (in part) caused by vibrational anharmonicity; although the processes of IR multiple photon excitation and emission are different, the bandwidths observed in the spectra are comparable (Oomens et al. 2003). The use of different ion sources and the capability of mass 
selection prior to spectroscopic interrogation makes this method very versatile, which is reflected in the variety of ionic PAH-related species that have been investigated, such as $\mathrm{Fe}^{+} \mathrm{PAH}$ complexes (Simon et al. 2008; Szczepanski et al. 2006) and protonated PAHs (Knorke et al. 2009; Lorenz et al. 2007; Vala et al. 2009b). We anticipate that mass spectrometry based spectroscopic methods will be ideally suited to investigate structures of PAH photo-products (Ekern et al. 1997; Jochims et al. 1994), which may provide important information to better understand the chemistry of PAHs in the diffuse ISM.

New technologies to record IR spectra of ionized species are continuously being explored and they may hold great promise for improved PAH ion spectra. Using various spectroscopic schemes, cryogenically cooled multipole ion traps have been recently applied to record spectra of cold gas-phase ions. Laser-induced reactions have been applied to obtain IR spectra of small hydrocarbon ions of astrophysical interest, including the intriguing $\mathrm{CH}_{5}^{+}$(Asvany et al. 2005) and hydronium (Asvany et al. 2008) ions. Cold ion traps have been applied to record electronic spectra of carbon chain ions of astrophysical interest (Rice et al. 2010). Combination of UV and IR lasers in cold ion traps to obtain IR hole-burning spectra of the cryogenically cooled ions has been applied to biomolecules of various sizes (Nagornova et al. 2010; Stearns et al. 2007). We anticipate that such methods will be applicable to PAH species as well.

Superfluid nanoscopic He droplets are known to provide an extremely noninteracting and cold $(0.4 \mathrm{~K})$ matrix to study the spectroscopy of molecular species (Goyal et al. 1992; Hartmann et al. 1996). Drabbels and coworkers are now applying these methods to study the spectroscopy of ions, including aromatic species, at high resolutions (Loginov et al. 2008; Smolarek et al. 2010).

\section{Concluding remarks}

In this review, I have given an overview of the various experimental methods that have been applied to obtain IR spectra of PAHs since the 1980's, when these species were originally hypothesized to occur abundantly in the ISM. The experimental IR spectra provide important benchmarks for theoretical investigations of the spectral properties of PAHs, which are discussed elsewhere in this volume. In addition, theory and experiments to understand PAH spectra in other spectral ranges, particularly in the $\mathrm{UV} /$ vis range are addressed elsewhere in this issue. We thus conclude that much progress has been made over the past 25 years in the experimental characterization of the spectra of PAHs, their ions and various of their derivatives.

\section{References}

Allamandola, L., Hudgins, D., \& Sandford, S., 1999, Astrophys. J., 511, L115

Allamandola, L., Tielens, A., \& Barker, J., 1985, Astrophys. J., 290, L25

Allamandola, L., Tielens, A., \& Barker, J., 1989, Astrophys. J. Suppl. Ser., 71, 733 
Alvaro Galué, H., Pirali, O., \& Oomens, J., 2010, Astron. Astrophys., 517, A15

Asvany, O., Ricken, O., Müller, H.S.P., et al., 2008, Phys. Rev. Lett., 100, 233004

Asvany, O., Schlemmer, S., Redlich, B., et al., 2005, Science 309, 1219

Barker, J., Allamandola, L., \& Tielens, A., 1987, Astrophys. J., 315, L61

Bauschlicher, C.W., Peeters, E., \& Allamandola, L.J., 2008, Astrophys. J., 678, 316

Bernstein, M.P., Sandford, S.A., Mattioda, A.L., \& Allamandola, L.J., 2007, Astrophys. J., 664, 1264

Black, J.G., Yablonovitch, E., Bloembergen, N., \& Mukamel, S., 1977, Phys. Rev. Lett., 38,1131

Bosenberg, W.R., \& Guyer, D.R., 1993, J. Opt. Soc. Am. B, 10, 17161722

Bouwman, J., Paardekooper, D.M., Cuppen, H.M., Linnartz, H., \& Allamandola, L.J., 2009, Astrophys. J., 700, 56

Colangeli, L., Mennella, V., Baratta, G.A., Bussoletti, E., \& Strazzulla, G., 1992, Astrophys. J., 396, 369

Cook, D., \& Saykally, R., 1998, Astrophys. J., 493, 793

Cook, D., Schlemmer, S., Balucani, N., et al., 1998, J. Phys. Chem. A, 102, 1465

Cook, D., Schlemmer, S., Balucani, N., et al., 1996, Nature, 380, 227

Douberly, G.E., Ricks, A.M., Schleyer, P.v.R., \& Duncan, M.A., 2008, J. Phys. Chem. A, 112,4869

Duncan, M.A., 2000, Int. J. Mass Spectrom., 200, 545

Ekern, S., Marshall, A., Szczepanski, J., \& Vala, M., 1997, Astrophys. J., 488, L39

Eyler, J.R., 2009, Mass Spectrom. Rev., 28, 448

Flickinger, G.C., Wdowiak, T.J., \& Gomez, P.L., 1991, Astrophys. J., 380, L43

Fulara, J., Jakobi, M., \& Maier, J., 1993, Chem. Phys. Lett., 211, 227

Goyal, S., Schutt, D.L., \& Scoles, G., 1992, Phys. Rev. Lett., 69, 933

Grant, E.R., Schulz, P.A., Sudbo, A.S., Shen, Y.R., \& Lee, Y.T., 1978, Phys. Rev. Lett., 40, 115

Hartmann, M., Miller, R.E., Toennies, J.P., \& Vilesov, A.F., 1996, Science, 272, 1631

Hudgins, D., \& Allamandola, L., 1995a, J. Phys. Chem., 99, 3033

Hudgins, D., \& Allamandola, L., 1995b, J. Phys. Chem., 99, 8978

Hudgins, D., Bauschlicher, C., \& Allamandola, L., 2005, Astrophys. J., 632, 316

Hudgins, D., Sandford, S., \& Allamandola, L., 1994, J. Phys. Chem., 98, 4243

Joalland, B., Simon, A., Marsden, C.J., \& Joblin, C., 2009, Astron. \& Astrophys., 494, 969

Joblin, C., Boissel, P., Leger, A., d'Hendecourt, L., \& Defourneau, D., 1995, Astron. Astrophys., 299, 835

Joblin, C., d'Hendecourt, L., Leger, A., \& Defourneau, D., 1994, Astron. Astrophys., 281,923

Jochims, H.W., Ruhl, E., Baumgartel, H., Tobita, S., \& Leach, S., 1994, Astrophys. J., 420, 307

Kim, H., \& Saykally, R., 2002, Astrophys. J. Suppl. Ser., 143, 455

Kim, H., \& Saykally, R., 2003, Rev. Sci. Instrum., 74, 2488

Kim, H., Wagner, D., \& Saykally, R., 2001, Phys. Rev. Lett., 86, 5691 
Klotz, A., Marty, P., Boissel, P., et al., 1996, Plan. Space Sci., 44, 957

Knorke, H., Langer, J., Oomens, J., \& Dopfer, O., 2009, Astrophys. J., 706, L66

Langhoff, S.R., 1996, J. Phys. Chem., 100, 2819

Léger, A., \& Puget, J., 1984, Astron. Astrophys., 137, L5

Lisy, J.M., 2006, J. Chem. Phys., 125, 132302

Loginov, E., Braun, A., \& Drabbels, M., 2008, Phys. Chem. Chem. Phys., 10, 6107

Lorenz, U.J., Solcà, N., Lemaire, J., Maitre, P., \& Dopfer, O., 2007, Angew. Chem. Int. Ed., 46, 6714

Malloci, G., Joblin, C., \& Mulas, G., 2007, Chem. Phys., 332, 353

Mattioda, A., Hudgins, D., Bauschlicher, C., Rosi, M., \& Allamandola, L., 2003, J. Phys. Chem. A, 107, 1486

Mattioda, A.L., Ricca, A., Tucker, J., Bauschlicher, Jr., C.W., \& Allamandola, L.J., 2009, Astron. J., 137, 4054

Moutou, C., Leger, A., \& dHendecourt, L., 1996, Astron. Astrophys., 310, 297

Nagornova, N.S., Rizzo, T.R., \& Boyarkin, O.V., 2010, J. Am. Chem. Soc., 132, 4040

Oepts, D., van der Meer, A.F.G., \& van Amersfoort, P.W., 1995, Infrared Phys. Technol., 36,297

Okumura, M., Yeh, L.I., \& Lee, Y.T., 1985, J. Chem. Phys., 83, 3705

Okumura, M., Yeh, L.I., Myers, J.D., \& Lee, Y.T., 1990, J. Phys. Chem., 94, 3416

Oomens, J., Sartakov, B.G., Tielens, A.G.G.M., Meijer, G., \& von Helden, G., 2001, Astrophys. J., 560, L99

Oomens, J., Tielens, A.G.G.M., Sartakov, B.G., von Helden, G., \& Meijer, G., 2003, Astrophys. J., 591, 968

Oomens, J., van Roij, A.J.A., Meijer, G., \& von Helden, G., 2000, Astrophys. J., 542, 404

O'Shea, P.G., \& Freund, H.P. 2001, Science, 292, 1853

Pauzat, F., Talbi, D., Miller, M.D., DeFrees, D.J., \& Ellinger, Y., 1992, J. Phys. Chem, 96,7882

Pech, C., Joblin, C., Boissel, P., 2002, Astron. Astrophys., 388, 639

Peeters, E., Hony, S., Van Kerckhoven, C., et al., 2002, Astron. Astrophys., 390, 1089

Piest, H., von Helden, G., \& Meijer, G., 1999a, Astrophys. J., 520, L75

Piest, H., von Helden, G., \& Meijer, G., 1999b, J. Chem. Phys., 110, 2010

Piest, J., Oomens, J., Bakker, J., von Helden, G., \& Meijer, G., 2001, Spectrochim. Acta A, 57,717

Pirali, O., \& Vervloet, M., 2006, Chem. Phys. Lett., 423, 376

Pirali, O., Vervloet, M., Mulas, G., Malloci, G., \& Joblin, C., 2009, Phys. Chem. Chem. Phys., 11, 3443

Rice, C.A., Rudnev, V., Dietsche, R., \& Maier, J.P., 2010, Astron. J., 140, 203

Ricks, A.M., Douberly, G.E., \& Duncan, M.A., 2009, Astrophys. J., 702, 301

Shan, J., Suton, M., \& Lee, L.C., 1991, Astrophys. J., 383, 459

Simon, A., Joblin, C., Polfer, N., \& Oomens, J., 2008, J. Phys. Chem. A, 112, 8551

Smolarek, S., Vdovin, A., Perrier, D.L., et al., 2010, J. Am. Chem. Soc., 132, 6315

Solcà, N., \& Dopfer, O., 2004, J. Am. Chem. Soc., 126, 1716 
Stearns, J.A., Mercier, S., Seaiby, C., et al., 2007, J. Am. Chem. Soc., 129, 11814

Szczepanski, J., Roser, D., Personette, W., et al., 1992, J. Phys. Chem., 96, 7876

Szczepanski, J., \& Vala, M., 1991, J. Phys. Chem., 95, 2792

Szczepanski, J., \& Vala, M., 1993a, Astrophys. J., 414, 646

Szczepanski, J., \& Vala, M., 1993b, Nature, 363, 699

Szczepanski, J., Wang, H., Vala, M., et al., 2006, Astrophys. J., 646, 666

Vala, M., Szczepanski, J., Dunbar, R., Oomens, J., \& Steill, J.D., 2009a, Chem. Phys. Lett., 473, 43

Vala, M., Szczepanski, J., Oomens, J., \& Steill, J.D., 2009b, J. Am. Chem. Soc., 131, 5784

Wang, Y., Szczepanski, J., \& Vala, M., 2007, Chem. Phys., 342, 107

Williams, R.M., \& Leone, S.R. 1995, Astrophys. J., 443, 675

Ysard, N., \& Verstraete, L. 2010, Astron. \& Astrophys., 509, A12 
